\title{
Hallazgos electroencefalográficos en familiares asintomáticos de pacientes con epilepsia mioclónica juvenil: su relación con el grado de parentesco
}

\author{
Iris E. Martínez-Juárez, ${ }^{1 *}$ Aurelio Jara-Prado, ${ }^{2}$ Adriana Ochoa-Morales, ${ }^{3}$ Nancy Castro-Veloz, ${ }^{4}$ \\ Andrea Santos-Peyret, ${ }^{1}$ Jimena Armenta-Báez ${ }^{1}$ y Mario A. Sebastián-Díaz ${ }^{5}$ \\ ${ }^{1}$ Instituto Nacional de Neurología y Neurocirugía "Manuel Velasco Suárez", Clínica de Epilepsia, Ciudad de México; ${ }^{2}$ Instituto Nacional de Neurología \\ y Neurocirugía "Manuel Velasco Suárez", Departamento de Neurogenética, Ciudad de México; ${ }^{3}$ nstituto Nacional de Neurología y Neurocirugía \\ "Manuel Velasco Suárez", Departamento de Neurogenética, Ciudad de México; "Instituto Mexicano del Seguro Social, Consulta Externa, Durango; \\ ${ }^{5}$ Universidad Anáhuac Norte, Facultad de Ciencias de la Salud, Estado de México. México
}

\section{Resumen}

Introducción: Se ha reportado de 1 a 71 \% de prevalencia de anormalidades en el electroencefalograma (EEG) de familiares asintomáticos de pacientes con epilepsia mioclónica juvenil (EMJ). Objetivo: Determinar la frecuencia de anormalidades en el EEG en familiares asintomáticos de pacientes con EMJ de acuerdo con el grado de parentesco. Métodos: Estudio prospectivo y analítico. Se incluyeron familiares de primer, segundo y tercer grado de pacientes con EMJ, quienes aceptaron participar y firmaron el consentimiento informado. El análisis fue descriptivo bivariado. Resultados: Se incluyeron 209 familiares asintomáticos, 115 (55\%) mujeres y 94 (45 \%) hombres, con edad media de $35.9 \pm 16.9$ (rango entre seis y 73 años); 44 familiares (21.1\%) tuvieron EEG anormal. Los familiares de primer grado (12\%) cursaron con mayor frecuencia con anormalidades en comparación con los de segundo y tercer grado $(p=0.007$ ). Conclusiones: Se observaron anormalidades en el EEG de una tercera parte de los familiares asintomáticos. Es importante resaltar que existieron más alteraciones entre los familiares de primer grado. En un futuro, estos hallazgos permitirán estimar el riesgo de desarrollar la enfermedad clínicamente y brindar consejo genético.

PALABRAS CLAVE: Electroencefalograma. Epilepsia mioclónica juvenil. Familiares asintomáticos.

\section{Electroencephalographic findings in asymptomatic relatives of patients with juvenile myoclonic epilepsy: relationship with the degree of kinship}

\begin{abstract}
Introduction: A prevalence of 1 to $71 \%$ of electroencephalogram (EEG) abnormalities has been reported in asymptomatic relatives of patients with juvenile myoclonic epilepsy (JME). Objective: To determine the frequency of EEG abnormalities in asymptomatic relatives of patients with JME according to the degree of kinship. Methods: Prospective, analytical study. First-, second- and third-degree relatives of patients with JME who agreed to participate and signed informed consent were included. The analysis was descriptive, bivariate. Results: 209 asymptomatic relatives were included, out of which 115 (55 \%) were females and 94 (45\%) were males, with a mean age of $35.9 \pm 16.9$ (range between 6 and 73 years). Forty-four (21.1\%) relatives had abnormal EEGs. First-degree relatives (12\%) had abnormalities more frequently in comparison with second-and third-degree relatives $(p=0.007)$. Conclusions: EEG abnormalities were observed in one third of asymptomatic relatives. It is important to highlight that there were more alterations among first-degree relatives. In the future, these findings might enable for the risk of clinically developing the disease to be estimated and for genetic counseling to be provided.
\end{abstract}

KEY WORDS: Electroencephalogram. Juvenile myoclonic epilepsy. Asymptomatic relatives.

Correspondencia:

*Iris E. Martínez-Juárez

E-mail: clinicadeepilepsia @innn.edu.mx
Fecha de recepción: 24-11-2020

Fecha de aceptación: 10-12-2020

DOI: 10.24875/GMM.20000856
Gac Med Mex. 2021;157:425-430

Disponible en PubMed

www.gacetamedicademexico.com

0016-3813/@ 2020 Academia Nacional de Medicina de México, A.C. Publicado por Permanyer. Este es un artículo open access bajo la licencia CC BY-NC-ND (http://creativecommons.org/licenses/by-nc-nd/4.0/). 


\section{Introducción}

La primera descripción de epilepsia mioclónica juvenil (EMJ) fue realizada en 1867 por Herpin, quien denominó como "tirones" a las sacudidas mioclónicas. Posteriormente, en 1957, Janz y Christian publicaron el artículo "Pequeño mal impulsivo". Delgado Escueta y Bacsal describieron la EMJ como epilepsia mioclónica de Janz. ${ }^{2}$

La EMJ es un síndrome epiléptico responsable de 6 a $12 \%$ de todas las epilepsias. ${ }^{3}$ Se ha estimado que su prevalencia es de $18 \%$ entre las epilepsias genéticas. ${ }^{4} \mathrm{La}$ edad de presentación es entre los seis y 22 años, pero $50 \%$ de los casos se presenta entre los 13 y 16 años. ${ }^{5-8}$ La EMJ comienza con crisis mioclónicas que ocurren usualmente después del despertar y son desencadenadas por deprivación del sueño y fatiga. Además de las mioclonías, $95 \%$ de los pacientes cursa con crisis tónico-clónicas generalizadas y una tercera parte también presenta crisis de ausencia. ${ }^{9}$ La etiología es genética, con una forma de herencia poligénica y evidencia de herencia autosómica dominante, autosómica recesiva y multifactorial..$^{5,10-13}$

Janz y Christian también reportaron que $41 \%$ de los familiares de pacientes tuvo epilepsia clínica, más frecuentemente las madres y las hijas. Existe una mayor incidencia de epilepsia en familiares de primer grado que en los de segundo grado en EMJ.14

Durón et al. ${ }^{9}$ encontraron anormalidades en el electroencefalograma de 24 familiares asintomáticos de pacientes con EMJ. De los 24 familiares, $63 \%$ presentó actividad epileptiforme caracterizada por complejos de polipunta onda de 4 a $6 \mathrm{~Hz} ; 17 \%$, disfunción focal o generalizada; $12 \%$, complejos punta onda de 3 a $5 \mathrm{~Hz}$; y $8 \%$, brotes de ondas agudas focales 0 generalizadas. La mayoría de los familiares asintomáticos con anormalidades en el electroencefalograma fue del sexo femenino.

Jayalakshmi et al..$^{14}$ evaluaron a 116 familiares asintomáticos de primer grado de pacientes con EMJ; encontraron que las anormalidades en el electroencefalograma fueron de actividad de polipunta onda/ onda lenta en $6 \%$ : dos sujetos mostraron un solo paroxismo, mientras cinco tuvieron múltiples paroxismos de complejos de polipunta onda/onda lenta, con frecuencias entre 4 y $5 \mathrm{~Hz}$; solo seis sujetos presentaron actividad theta intermitente. También encontraron que la incidencia de epilepsia entre los familiares de primer grado de pacientes con EMJ fue de $38.7 \% .{ }^{14}$
Los familiares de pacientes con EMJ pueden tener anormalidades electroencefalográficas y cursar asintomáticos. Estos hallazgos permiten entender la transmisión genética de la enfermedad, ya que los familiares, particularmente los de primer grado, pueden ser portadores asintomáticos de la mutación y solo mostrar alteraciones en el electroencefalograma.

El presente estudio describe la frecuencia de alteraciones electroencefalográficas en familiares asintomáticos de primer, segundo y tercer grado de pacientes con EMJ en una cohorte de pacientes del Instituto Nacional de Neurología y Neurocirugía "Manuel Velasco Suárez" en la Ciudad de México. Esta cohorte se podrá seguir en el tiempo y determinar qué familiares asintomáticos con anormalidades en el electroencefalograma desarrollarán manifestaciones clínicas de la enfermedad.

\section{Métodos}

Se realizó un estudio observacional, prospectivo, transversal, analítico, que incluyó consecutivamente a familiares de primer, segundo y tercer grado de pacientes con EMJ atendidos en el Instituto Nacional de Neurología "Manuel Velasco Suárez" de enero de 2008 al momento de este reporte quienes aceptaron participar en el estudio, el cual cuenta con la aprobación de los comités de bioética e investigación del Instituto.

Se incluyeron familiares asintomáticos de pacientes con EMJ atendidos en la consulta externa de la clínica de epilepsia, en función de la decisión voluntaria de participar tras informarles de la posibilidad de hacerlo. La participación no fue remunerada y los participantes o sus tutores, en los menores de edad, firmaron un consentimiento $o$ asentimiento informado, respectivamente. Posteriormente, se recabó la información relativa a los datos personales y se realizó un electroencefalograma de 20 minutos de duración.

Se incluyeron familiares con una edad mayor de cinco años. Se excluyeron los sujetos con historia de enfermedad sistémica o neurológica relevante que pueda modificar el electroencefalograma, con diagnóstico definido de epilepsia, uso de fármacos antiepilépticos u otros medicamentos que modifican los resultados electroencefalográficos.

\section{Registro del electroencefalograma}

Los electroencefalogramas fueron revisados por los dos epileptólogos autores del estudio; los hallazgos se clasificaron de la siguiente manera: 
- Actividad de base: descripción de la frecuencia de base, la cual en adultos debe ser en rango alfa; asimismo, se debe describir la reactividad y simetría.

- Estimulación luminosa intermitente: respuesta fotoparoxística consistente en descargas occipitales de punta, punta onda o polipunta onda que se presentan durante el estímulo fótico.

- Disfunción cerebral: enlentecimiento difuso o focal del ritmo de base en rango alfa. Deben agregarse las áreas que puedan presentar un nivel de asimetría, tanto de frecuencia como de amplitud generalizada o focal por lóbulos (frontal, parietal, temporal y occipital).

- Actividad epiléptica: presencia de grafoelementos que se han descrito como epileptiformes, como las puntas y ondas agudas que se asocian generalmente con una onda lenta que las sigue y que pueden presentar variaciones como los complejos punta onda aguda, punta onda lenta o polipuntas.

Las características electroencefalográficas de las descargas están bien definidas e incluyen carácter paroxístico, morfología aguda, polaridad negativa, campo de distribución fisiológica y, generalmente, aparición de onda lenta después del elemento agudo. Solo la conjunción de estos elementos permite determinar la naturaleza epileptogénica. Los grafoelementos epileptiformes pueden ser generalizados, focales o multifocales. Las distintas distribuciones topográficas se correlacionan con los distintos tipos de epilepsia: primariamente generalizada o focal, generalizada o focal por lóbulos.

Los grafoelementos consisten en punta, polipunta, onda aguda, onda lenta, complejo punta onda lenta y complejo de polipunta onda lenta.

\section{Análisis estadístico}

Para el análisis de datos se elaboraron hojas de captura de datos y se realizó una base en el programa SPSS versión 15 (SPSS Inc., Chicago, Estados Unidos). Se realizó un análisis descriptivo con medias y desviación estándar de las variables numéricas y porcentajes de las variables nominales. Posteriormente se realizó un análisis univariado con el que se compararon los hallazgos de los electroencefalogramas de los familiares de primer, segundo y tercer grado mediante prueba de $\chi^{2} 0$ exacta de Fisher. Para fines del análisis se consideraron a los padres como familiares de primer

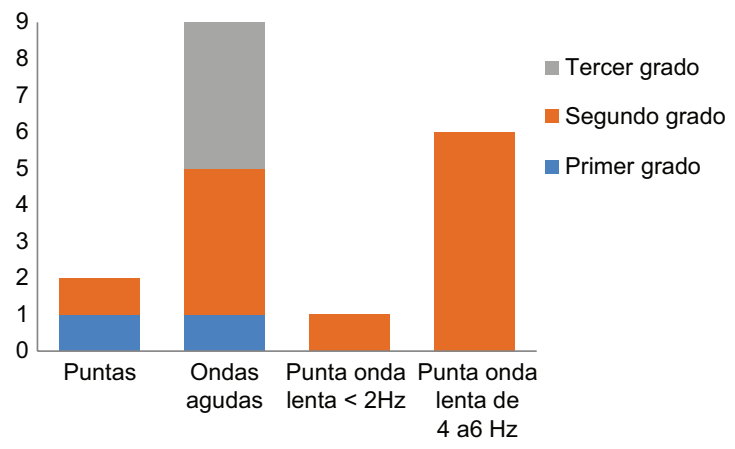

Figura 1. Tipo de grafoelementos epileptiformes en familiares asintomáticos de pacientes con epilepsia mioclónica juvenil de acuerdo con el grado de parentesco.

grado, a hermanos e hijos como familiares de segundo grado y a tíos, sobrinos y primos como familiares de tercer grado.

\section{Resultados}

Se incluyeron 209 familiares asintomáticos, todos cumplieron con los criterios de inclusión; 115 (55\%) fueron mujeres y 94 (45\%) hombres, con una media de edad de $35.9 \pm 16.9$ y un rango de seis a 73 años. En relación con la distribución por grado de parentesco se encontró que 89 (42.6\%) fueron de primer grado, 81 (38.8\%) de segundo grado y $39(18.7 \%)$ de tercer grado.

El electroencefalograma fue anormal en 44 familiares asintomáticos $(21.1 \%)$. De los familiares evaluados, ninguno presentó respuesta fotoconvulsiva 0 fotoparoxística. Los que presentaron mayor frecuencia de electroencefalogramas anormales fueron los familiares de segundo grado (Tabla 1).

De los 44 familiares con anormalidades, 28 (63.6\%) presentaron disfunción y 25 (56.8\%), actividad epiléptica. La actividad epiléptica generalizada más frecuente fue la presencia de complejos de punta onda lenta de 4 a $6 \mathrm{~Hz}$. Los hallazgos detallados del electroencefalograma se describen en la Tabla 1 y Figura 1.

En relación con el grado de parentesco y la presencia de alteraciones en el electroencefalograma, se encontró mayor afectación en los familiares de segundo grado ( $n=25,56.8 \%$ ), seguidos por los familiares de primer grado $(n=10,22.7 \%)$ y de tercer grado $(n=9,29.5 \%)$.

En el análisis bivariado para determinar la relación entre las anormalidades en el electroencefalograma 
Tabla 1. Hallazgos anormales electroencefalográficos en familiares asintomáticos de pacientes con epilepsia mioclónica juvenil

\begin{tabular}{|c|c|c|c|c|c|c|c|c|}
\hline \multirow[t]{2}{*}{ Hallazgo } & \multirow[t]{2}{*}{ Afectación } & \multicolumn{2}{|c|}{ Primer grado } & \multicolumn{2}{|c|}{ Segundo grado } & \multicolumn{2}{|c|}{ Tercer grado } & \multirow{2}{*}{$\frac{\text { Total }}{n}$} \\
\hline & & n & $\%$ & $\mathrm{n}$ & $\%$ & n & $\%$ & \\
\hline Electroencefalograma anormal & Total & 10 & 22.7 & 25 & 56.8 & 9 & 29.5 & 44 \\
\hline Disfunción & Total & 7 & 25 & 16 & 57 & 5 & 18 & 28 \\
\hline \multirow[t]{5}{*}{ Disfunción focal } & Total & 7 & 29.2 & 12 & 50 & 5 & 20.8 & 24 \\
\hline & $\begin{array}{l}\text { Frontal } \\
\text { Unilateral } \\
\text { Bilateral }\end{array}$ & $\begin{array}{l}1 \\
2\end{array}$ & $\begin{array}{l}25 \\
25\end{array}$ & $\begin{array}{l}2 \\
3\end{array}$ & $\begin{array}{c}59 \\
37.5\end{array}$ & $\begin{array}{l}1 \\
3\end{array}$ & $\begin{array}{c}25 \\
37.5\end{array}$ & $\begin{array}{l}4 \\
8\end{array}$ \\
\hline & $\begin{array}{l}\text { Temporal } \\
\text { Unilateral } \\
\text { Bilateral }\end{array}$ & $\begin{array}{l}2 \\
0\end{array}$ & $\begin{array}{c}28.6 \\
0\end{array}$ & $\begin{array}{l}4 \\
0\end{array}$ & $\begin{array}{c}57.1 \\
0\end{array}$ & $\begin{array}{l}1 \\
0\end{array}$ & $\begin{array}{c}14.3 \\
0\end{array}$ & $\begin{array}{l}7 \\
0\end{array}$ \\
\hline & $\begin{array}{l}\text { Parietal } \\
\text { Unilateral } \\
\text { Bilateral }\end{array}$ & $\begin{array}{l}0 \\
0\end{array}$ & $\begin{array}{l}0 \\
0\end{array}$ & $\begin{array}{l}0 \\
2\end{array}$ & $\begin{array}{c}0 \\
100\end{array}$ & $\begin{array}{l}0 \\
0\end{array}$ & $\begin{array}{l}0 \\
0\end{array}$ & $\begin{array}{l}0 \\
2\end{array}$ \\
\hline & $\begin{array}{l}\text { Occipital } \\
\text { Unilateral } \\
\text { Bilateral }\end{array}$ & $\begin{array}{l}0 \\
2\end{array}$ & $\begin{array}{c}0 \\
100\end{array}$ & $\begin{array}{l}1 \\
0\end{array}$ & $\begin{array}{c}100 \\
0\end{array}$ & $\begin{array}{l}0 \\
0\end{array}$ & $\begin{array}{l}0 \\
0\end{array}$ & $\begin{array}{l}1 \\
2\end{array}$ \\
\hline Disfunción generalizada & & 0 & 0 & 4 & 100 & 0 & 0 & 4 \\
\hline Actividad epiléptica & Total & 4 & 16 & 14 & 56 & 7 & 28 & 25 \\
\hline \multirow[t]{5}{*}{ Actividad epiléptica focal } & Total & 3 & 17.7 & 8 & 47 & 6 & 35.3 & 17 \\
\hline & $\begin{array}{l}\text { Frontal } \\
\text { Unilateral } \\
\text { Bilateral }\end{array}$ & $\begin{array}{l}0 \\
0\end{array}$ & $\begin{array}{l}0 \\
0\end{array}$ & $\begin{array}{l}2 \\
2\end{array}$ & $\begin{array}{l}50 \\
50\end{array}$ & $\begin{array}{l}2 \\
2\end{array}$ & $\begin{array}{l}50 \\
50\end{array}$ & $\begin{array}{l}4 \\
4\end{array}$ \\
\hline & $\begin{array}{l}\text { Temporal } \\
\text { Unilateral } \\
\text { Bilateral }\end{array}$ & $\begin{array}{l}1 \\
0\end{array}$ & $\begin{array}{c}25 \\
0\end{array}$ & $\begin{array}{l}2 \\
0\end{array}$ & $\begin{array}{c}50 \\
0\end{array}$ & $\begin{array}{l}1 \\
1\end{array}$ & $\begin{array}{c}25 \\
100\end{array}$ & $\begin{array}{l}4 \\
1\end{array}$ \\
\hline & $\begin{array}{l}\text { Parietal } \\
\text { Unilateral } \\
\text { Bilateral }\end{array}$ & $\begin{array}{l}0 \\
0\end{array}$ & $\begin{array}{l}0 \\
0\end{array}$ & $\begin{array}{l}0 \\
1\end{array}$ & $\begin{array}{c}0 \\
100\end{array}$ & $\begin{array}{l}0 \\
0\end{array}$ & $\begin{array}{l}0 \\
0\end{array}$ & $\begin{array}{l}0 \\
1\end{array}$ \\
\hline & $\begin{array}{l}\text { Occipital } \\
\text { Unilateral } \\
\text { Bilateral }\end{array}$ & $\begin{array}{l}1 \\
1\end{array}$ & $\begin{array}{l}50 \\
50\end{array}$ & $\begin{array}{l}1 \\
0\end{array}$ & $\begin{array}{c}50 \\
0\end{array}$ & $\begin{array}{l}0 \\
0\end{array}$ & $\begin{array}{l}0 \\
0\end{array}$ & $\begin{array}{l}2 \\
1\end{array}$ \\
\hline Actividad epiléptica generalizada & & 1 & 12.5 & 6 & 75 & 1 & 12.5 & 8 \\
\hline
\end{tabular}

y el grado de parentesco de los familiares asintomáticos, se encontró diferencia significativa en la presencia de un electroencefalograma anormal en familiares de segundo grado $(p=0.007)$.

\section{Discusión}

En este estudio se presentan los resultados de los hallazgos electroencefalográficos en familiares asintomáticos de primer, segundo y tercero grado de pacientes con EMJ. El presente estudio constituye el primero de este tipo realizado en México. Se encontraron anormalidades en el electroencefalograma hasta en una cuarta parte $(21.1 \%)$ de los familiares asintomáticos. Un metaanálisis reciente, que incluyó seis estudios con un total de 108 pacientes con EMJ y 206 familiares de primer grado, encontró que hasta $19 \%$ tenía anormalidades en el electroencefalograma. ${ }^{15}$

Cabe resaltar que la incidencia de actividad epileptiforme en el electroencefalograma de rutina puede ser de hasta $0.5 \%$ en adultos sanos. ${ }^{16} \mathrm{En}$ este estudio, hasta $21.1 \%$ de los familiares asintomáticos mostró anormalidades, proporción más alta que la encontrado en estudios de voluntarios sanos 
y en una investigación realizada en la India y en el metaanálisis mencionado. ${ }^{14,15} \mathrm{Al}$ realizar el análisis de asociación entre el grado de parentesco y los hallazgos anormales en el electroencefalograma, se encontraron diferencias significativas: las anormalidades fueron frecuentes en familiares de primer grado seguidos de los de segundo y tercer grado. En estudios moleculares de penetrancia se ha registrado que a mayor distancia del paciente afectado, menor el riesgo de epilepsia. Análisis previos han descrito que existe un mayor riesgo de desarrollar epilepsia genética generalizada en miembros de la familiar nuclear. ${ }^{17,18}$

Con pruebas neuropsicológicas a pacientes con EMJ y sus hermanos se han encontrado anormalidades en ambos. ${ }^{19}$ En un estudio se evidenció que los pacientes con EMJ y sus hermanos asintomáticos muestran alteraciones en las pruebas de función ejecutiva, como la memoria prospectiva, en relación con individuos sanos; se concluyó que las disfunciones frontales también pueden estar genéticamente determinadas en pacientes y familiares sanos. ${ }^{20}$ Sería interesante indagar si esto tiene relación con la presencia o ausencia de anormalidades en el electroencefalograma en los familiares de los pacientes con EMJ.

Entre las limitaciones del estudio está el tamaño de la muestra, ya que aun cuando el número de sujetos analizados fue adecuado, las anormalidades solo se encontraron en una cuarta parte, por lo que los resultados pudieran ser más concluyentes con una muestra mayor. Por lo anterior, no fue posible realizar un cálculo de riesgo, que pudiera enriquecer los hallazgos.

La presente investigación constituye la base para desarrollar estudios de seguimiento de los individuos con anormalidades en el electroencefalograma, en quienes se realice determinación molecular de EMJ y se defina cuántos de ellos presentarán manifestaciones clínicas de epilepsia genética en alguna etapa de la vida.

\section{Conclusiones}

En el presente estudio se observó la presencia de anormalidades electroencefalográfica en casi una cuarta parte de los familiares asintomáticos de pacientes con EMJ. Es importante resaltar que se observaron más alteraciones en los familiares de segundo grado.
Estos hallazgos confirman la transmisión genética de la EMJ, por lo que es importante hacer énfasis en la evaluación subclínica o clínica de los familiares de los pacientes con esta enfermedad. En un futuro quizás estos hallazgos anormales permitan estimar adecuadamente el riesgo de desarrollar la enfermedad clínicamente y realizar un consejo genético.

\section{Agradecimientos}

Los autores agraceden a los pacientes y familiares que aceptaron participar en este estudio.

\section{Conflicto de intereses}

Los autores declaran no tener conflicto de intereses alguno.

\section{Financiamiento}

La presente investigación no recibió ninguna beca específica de agencias de los sectores públicos, comercial, o sin ánimo de lucro.

\section{Responsabilidades éticas}

Protección de personas y animales. Los autores declaran que los procedimientos seguidos se conformaron a las normas éticas del comité de experimentación humana responsable y de acuerdo con la Asociación Médica Mundial y la Declaración de Helsinki.

Confidencialidad de los datos. Los autores declaran que siguieron los protocolos de su centro de trabajo sobre la publicación de datos de pacientes.

Derecho a la privacidad y consentimiento informado. Los autores obtuvieron el consentimiento informado de los pacientes o sujetos referidos en el artículo. Este documento obra en poder del autor de correspondencia.

\section{Bibliografía}

1. Renganathan R, Delanty N. Juvenile myoclonic epilepsy: under-appreciated and under-diagnosed. Postgrad Med J. 2003;79:78-80.

2. Delgado-Escueta A, Enrile-Bacsal F. Juvenile myoclonic epilepsy of Janz. Neurology. 1984;34:285-294.

3. Martínez-Juárez IE, Alonso ME, Medina MT, Durón RM, Bailey JN, López-Ruiz M, et al. Juvenile myoclonic epilepsy subsyndromes: family studies and long-term follow-up. Brain. 2006;129:1269-1280.

4. Camfield CS, Striano P, Camfield PR. Epidemiology of juvenile myoclonic epilepsy. Epilepsy Behav. 2013;28:S15-S7.

5. Alfradique I, Vasconcelos MM. Juvenile myoclonic epilepsy. Arq Neuropsiquiatr. 2007:65:1266-1271.

6. Alonso ME, Medina MT, Martínez-Juárez IE, Durón RM, Bailey JN, López-Ruiz M, et al. Familial juvenile myoclonic epilepsy. Adv Neurol. 2005;95:227. 
7. Kleveland G, Engelsen BA. Juvenile myoclonic epilepsy: clinical characteristics, treatment and prognosis in a Norwegian population of patients. Seizure. 1998;7:31-38

8. Kasteleijn-Nolst-Trenite DGA, Schmitz B, Janz D, Delgado-Escueta AV Thomas $\mathrm{P}$. Hirsch $\mathrm{E}$, et al. Consensus on diagnosis and management of JME: From founder's observations to current trends. Epilepsy Behav. 2013;28:S87-S90.

9. Durón RM, Medina MT, Martínez-Juárez IE, Bailey JN, Perez-Gosiengfiao KT, Ramos-Ramírez R, et al. Seizures of idiopathic generalized epilepsies. Epilepsia. 2005;46:34-47.

10. Bailey JN, Patterson C, de Nijs L, Durón RM, Nguyen V-H, Tanaka M, et al. EFHC1 variants in juvenile myoclonic epilepsy: reanalysis according to NHGRI and ACMG guidelines for assigning disease causality. Genet Med. 2017;19:144-156.

11. Bailey JN, de Nijs L, Bai D, Suzuki T, Miyamoto H, Tanaka M, et al Variant intestinal-cell kinase in juvenile myoclonic epilepsy. $\mathrm{N}$ Engl $J$ Med. 2018;378:1018-1028

12. Medina M, Suzuki T, Alonso M, Durón R, Martínez-Juárez I, Bailey J, et al. Novel mutations in Myoclonin1/EFHC1 in sporadic and familial juvenile myoclonic epilepsy. Neurology. 2008;70:2137-2144

13. Suzuki T, Delgado-Escueta AV, Aguan K, Alonso ME, Shi J, Hara Y et al. Mutations in EFHC1 cause juvenile myoclonic epilepsy. Nat Genet. 2004;36:842-849
14. Jayalakshmi SS, Mohandas S, Sailaja S, Borgohain R. Clinical and electroencephalographic study of first-degree relatives and probands with juvenile myoclonic epilepsy. Seizure. 2006;15:177-183.

15. Tashkandi M, Baarma D, Tricco AC, Boelman C, Alkhater $R$, Minassian BA. EEG of asymptomatic first-degree relatives of patients with juvenile myoclonic, childhood absence and rolandic epilepsy: a systematic review and meta-analysis. Epileptic Disord. 2019;21:30-41.

16. Smith S. EEG in the diagnosis, classification, and management of patients with epilepsy. J Neurol Neurosurg Psychiatry. 2005;76: ii2-ii7

17. Peljto AL, Barker-Cummings C, Vasoli VM, Leibson CL, Hauser WA, Buchhalter JR, et al. Familial risk of epilepsy: a population-based study. Brain. 2014;137:795-805

18. Bianchi A, Viaggi S, Chiossi E, LICE Episcreen Group. Family study of epilepsy in first degree relatives: data from the Italian Episcreen Study. Seizure. 2003;12:203-210.

19. Iqbal N, Caswell HL, Hare DJ, Pilkington O, Mercer S, Duncan S. Neuropsychological profiles of patients with juvenile myoclonic epilepsy and their siblings: a preliminary controlled experimental video-EEG case series. Epilepsy Behav. 2009;14:516-521.

20. Wandschneider B, Kopp U, Kliegel M, Stephani U, Kurlemann G, Janz D, et al. Prospective memory in patients with juvenile myoclonic epilepsy and their healthy siblings. Neurology. 2010;75:2161-2167. 